\title{
The Effectiveness of Acceptance and Commitment-Based Therapy on Assertiveness of Imposed War Veterans' daughters
}

\section{ART ICLE INF O}

\section{Article Type}

Original Research

\section{Authors}

Hadyian S. ${ }^{1} M S C$,

Dehghani A. ${ }^{* 1} P h D$

How to cite this article
Hadyian S, Dehghani A. The Effe-
ctiveness of Acceptance and Co-
mmitment-Based Therapy on Ass-
ertiveness of Imposed War Vete-
rans' daughters. Journal of War \&
Public Health.2019; $11(3): 147-151$.

${ }^{1}$ Clinical Psychology Department, Humanities Faculty, Najafabad Branch, Islamic Azad University, Najafabad, Iran

\section{*Correspondence}

Address: Clinical Psychology Department, Humanities Faculty, Najafabad Branch, Islamic Azad University, Najafabad, Iran. Postal Code: 8514143131

Phone: +98 (31) 32404263

Fax: +98 (31) 42291016

dehghani2016@phu.iaun.ac.ir

\section{Article History}

Received: November 6, 2018

Accepted: May 15, 2019

ePublished: July 21, 2019

\section{A B S T R A C T}

Objectives The consequences of war are profound, complex, and continuous and not only stopped by suffering the survived individuals of war from physical and mental disorders or damages, but also penetrate to their families and caregivers. The objective of the present study is to study the effectiveness of acceptance and commitment based therapy on the assertiveness of imposed war veterans' daughters.

Materials \& Methods This is semi-experimental research (pretest-posttest with a control group) performed on veterans' daughters' population of Falavarjan town in 2017. 30 individuals were selected using Purposive Sampling method and randomly assigned in two test and control groups (15 individuals for each group). The Test group received acceptance and commitment based therapy during 8 sessions, but the control group did not receive any training. Assertiveness Questionnaire of Gmbryl and Ritchie was used as a research tool. The data were analyzed using SPSS 23 software through single variable covariance, independent sample T-test, and chi-square test.

Findings In post-test phase, the mean of assertiveness scores in the test group had a significant difference compared to the control group $(\mathrm{p}<0.05)$ and the mean of scores in the test group was more than the control group.

Conclusion Acceptance and commitment based therapy is effective on the assertiveness of imposed war veterans' daughters.

Keywords Acceptance and Commitment Based Therapy; Assertiveness; Veterans

\section{I T A T I O N L I N K S}

[1] Veterans' mental health in the wake ... [2] The survey of veterans' depression and anxiety and its relationship ... [3] The prevalence of anxiety and depression disorders in the children of veterans of ... [4] Personal well-being and stress symptoms in wives of Iranian ... [5] Aggression, anxiety and social development in adolescent children of war ... [6] Evaluation of Mental health state in veterans family ... [7] Comparison of high school students mental health in regard ... [8] Problem solving as reinforcement in adolescent drug use ... [9] The effect of self-differentiation therapy on assertiveness ... [10] The effectiveness of an ACT informed intervention for managing ... [11] Acceptance and commitment ... [12] The application of acceptance and commitment therapy ... [13] Psychosocial treatment for methamphetamine use disorders: a ... [14] Depression, low self-esteem and ... [15] The effect of mindfulness training (MBSR) on the ... [16] The efficacy of mindfulness based stress reduction on mindfulness and assertiveness ... [17] Investigating the effectiveness of mindfulness-based cognitive therapy to reduce shyness and ... [18] Effectiveness of acceptance and commitment therapy on sexual function ... [19] Comparison of the efficacy of acceptance and ... [20] Putting the 'app' in happiness: a randomized controlled ... [21] The effectiveness of acceptance and commitment ... [22] The effectiveness of group-based acceptance and commitment ... [23] Flourishing in people with depressive symptomatology ... [24] Acceptance and commitment therapy for ... [25] Acceptance and commitment therapy for ... [26] An assertion inventory for use in assessment and ... [27] Relationship among assertiveness, self-esteem ... [28] Practical guide for therapists in acceptance and ... [29] Using acceptance and commitment therapy ... [30] Acceptance and commitment therapy, relational frame theory ... [31] A preliminary trial of twelve-step facilitation and acceptance and commitment ... 
حقوق ديكران همراه با جراتمندى اشاره دارد و بهعنوان توانايى تهايى

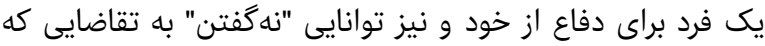

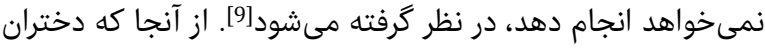

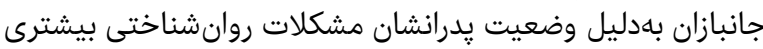

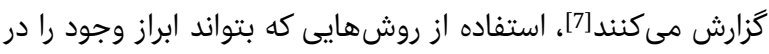

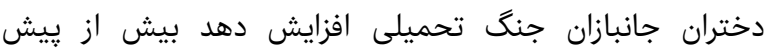

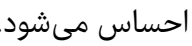

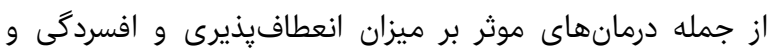

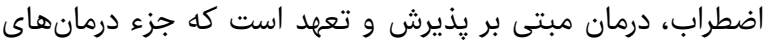

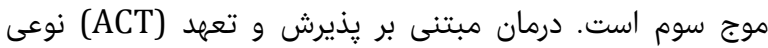

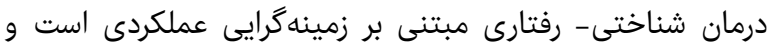

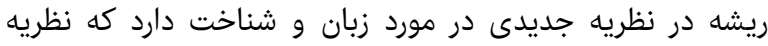

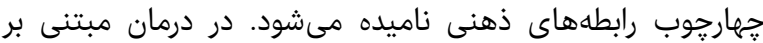

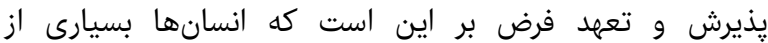

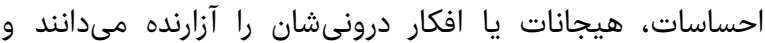

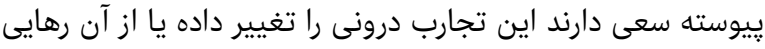

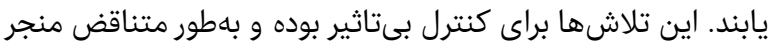

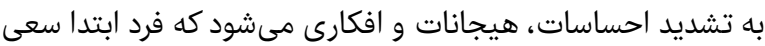

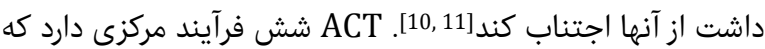

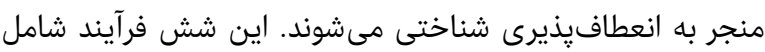

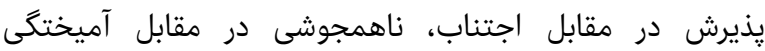

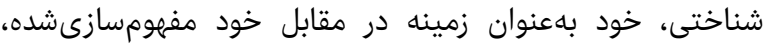
ارتباط زمان حال در مقابل غلبه كذشتنه و آينده مفهومسازى شيده،

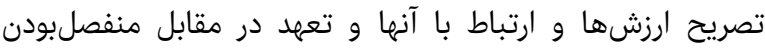

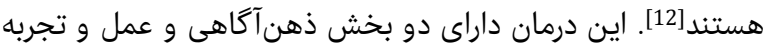

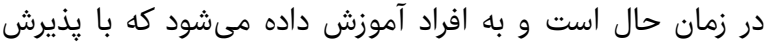

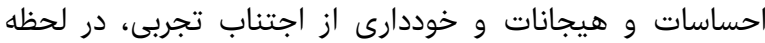

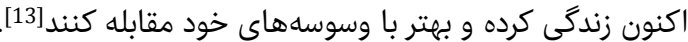

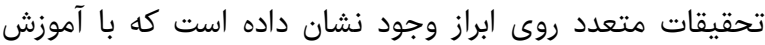

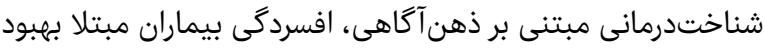

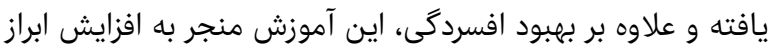

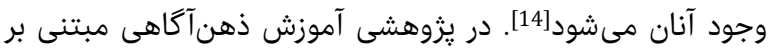

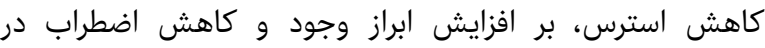

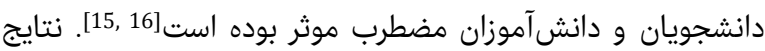

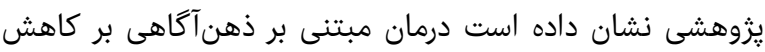

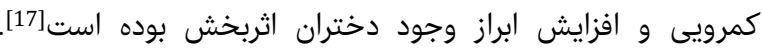

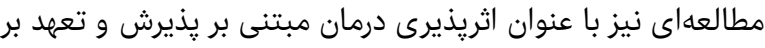

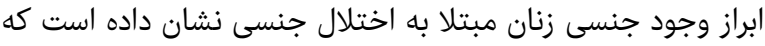

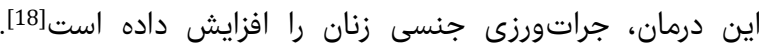

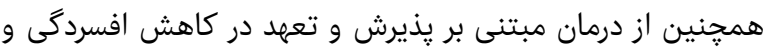

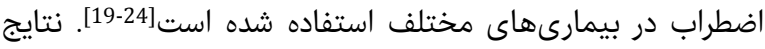

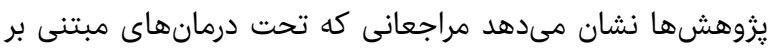

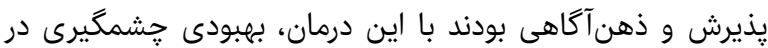

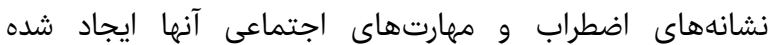

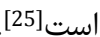

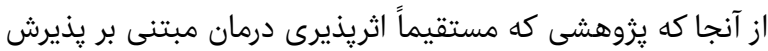

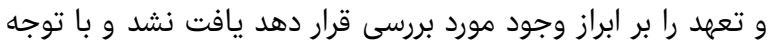

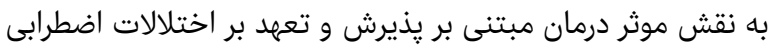

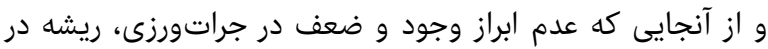

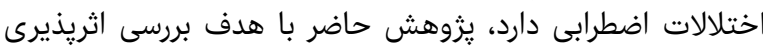

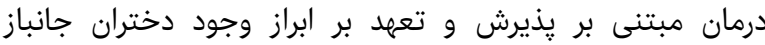

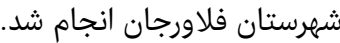

اثربخشى درمان مبتنى بر هذيرش و و تعهل بران بر ابراز وجود دختران جانبازان جنگ تحميلى لئيلى

صروهيه هاديان MSc

اكرم دهقانى * PhD

كَروه روانشناسى بالينى، دانشكده علوم انسانى، واحد نجفآباد، دانشكاه آزاد

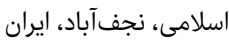

جكيده

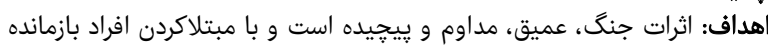

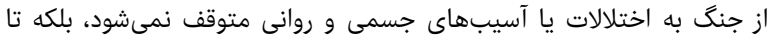

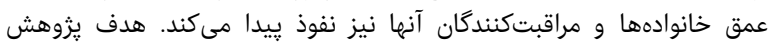

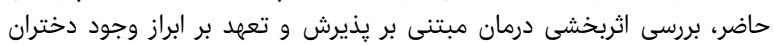

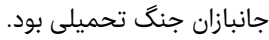

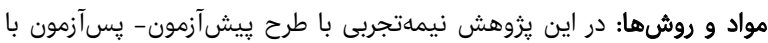

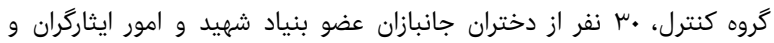

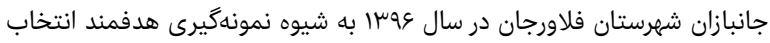

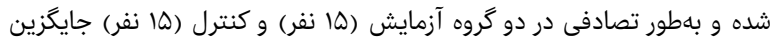

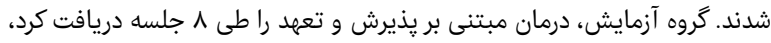

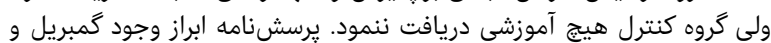

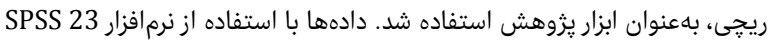
و از طريق آزمونهاى تحليل كوواريانس تكمتغيره، T مستقل و مجذور كاى الثراي تجزيه و تحليل شدند.

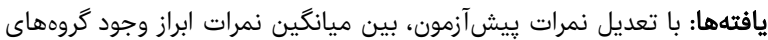

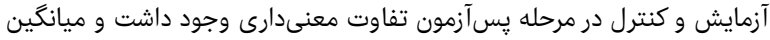

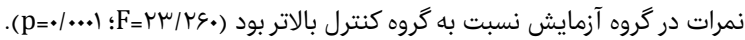

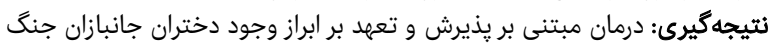
تحميلى موثر است دمان. كليدواثهما: درمان مبتنى بر يذيرش و تعهد، ابراز وجود، جانبازان

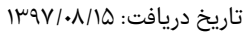

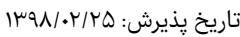

dehghani2016@phu.iaun.ac.ir :نويسنده مسئول:

مقدمه م

يكى از عوامل آسيبرسان به ساختار خانواده، معلوليت يكى از ازئ إنوان

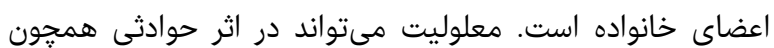

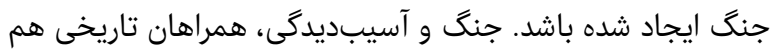

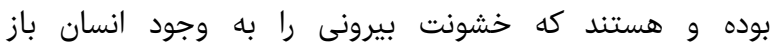

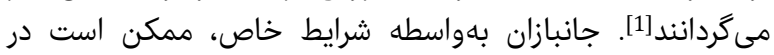

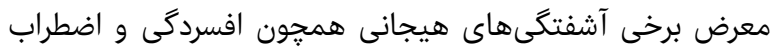

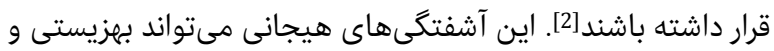

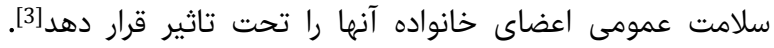

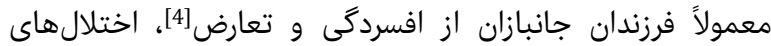

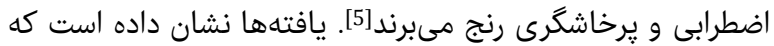

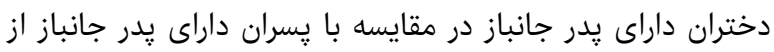

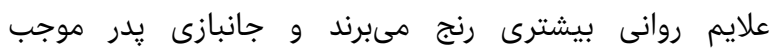

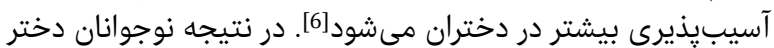

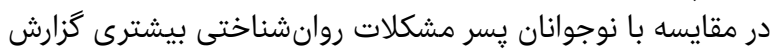

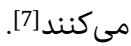

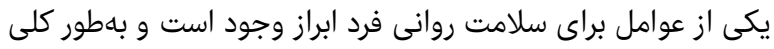

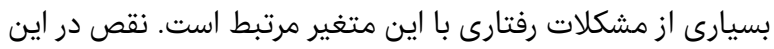

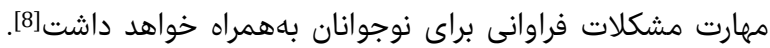

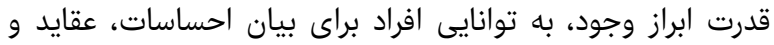

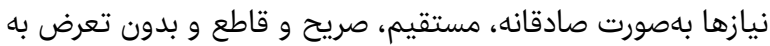




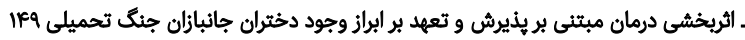

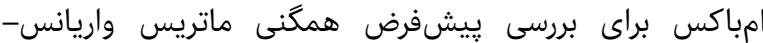

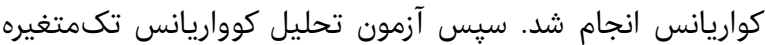

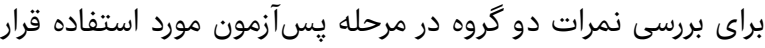

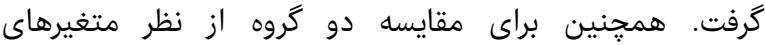

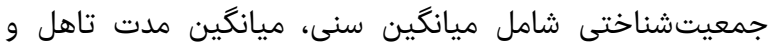

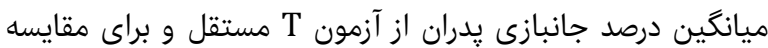

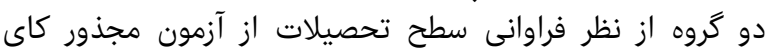

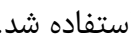

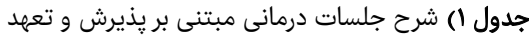

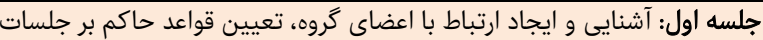

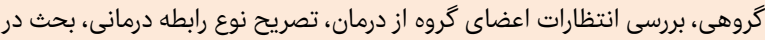

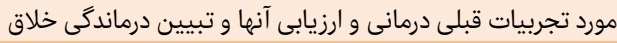

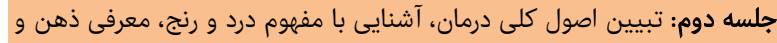

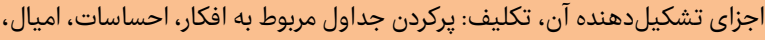

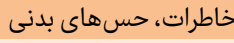

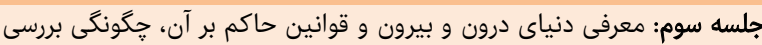

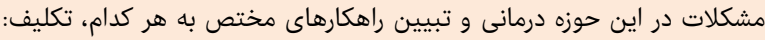

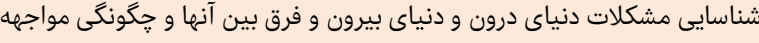
با هر كدام

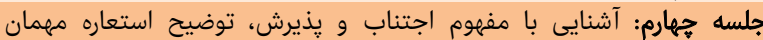

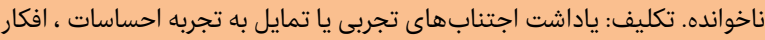

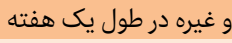

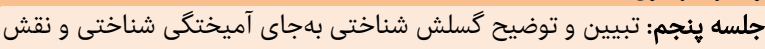

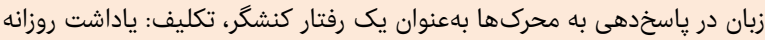

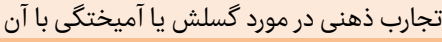

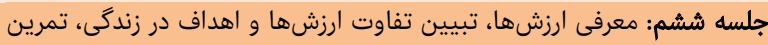

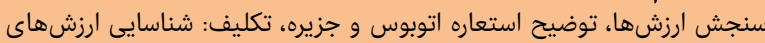
شخصى و اولويتبندى آنها تونها

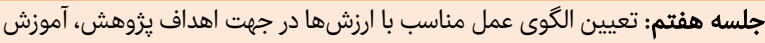

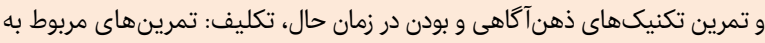

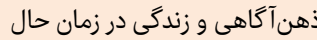

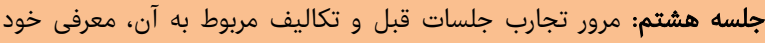

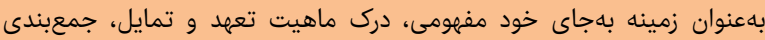
مطالب درمان در راه رسيدن بهاي ابراز وجودي دودي

يافتهها

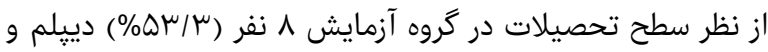

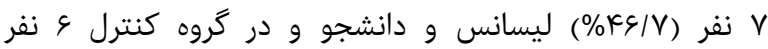

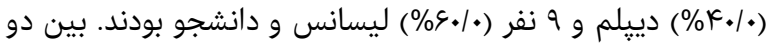

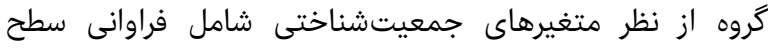

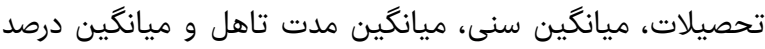

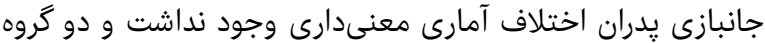

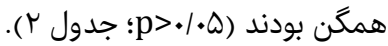

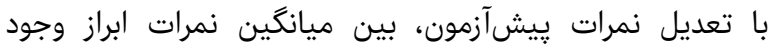

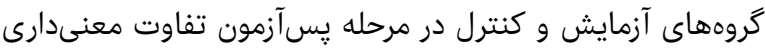

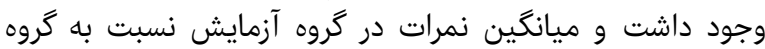

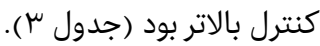

جدول r آميانكَين آمارى مربوط به متغيرهاى جمعيتشناختى در دو كروه

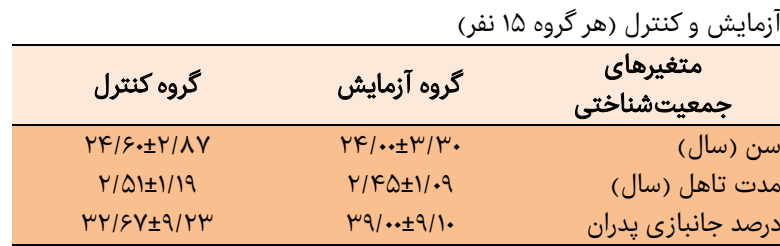

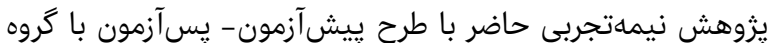

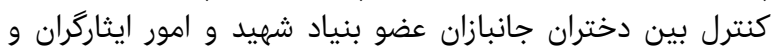

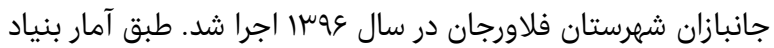

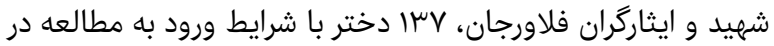

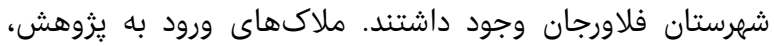

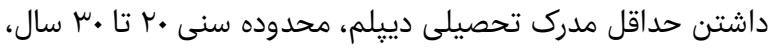

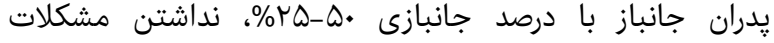

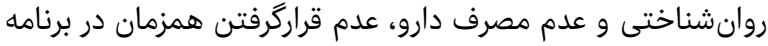

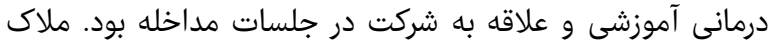

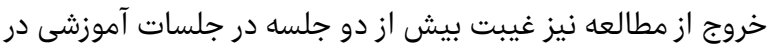

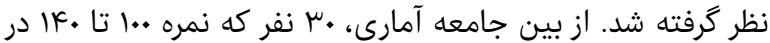

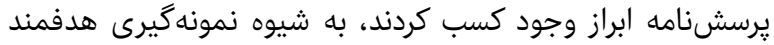

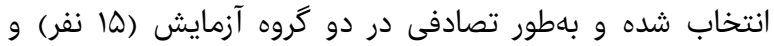

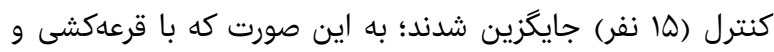

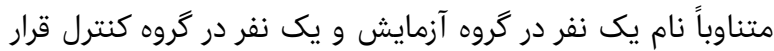

كرفت.

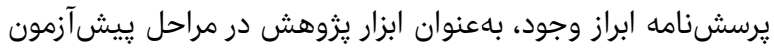

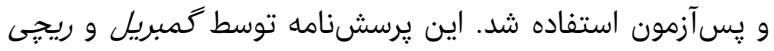

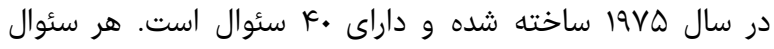

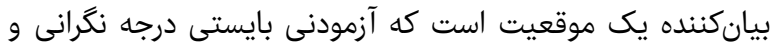

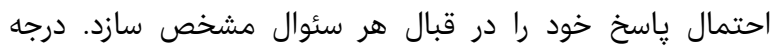

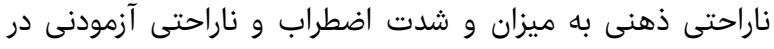

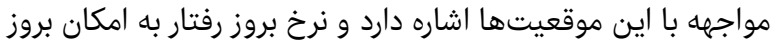

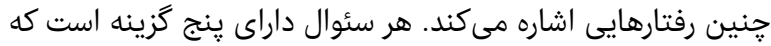

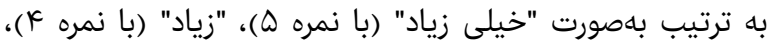

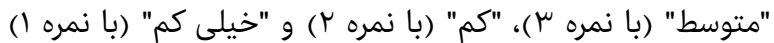

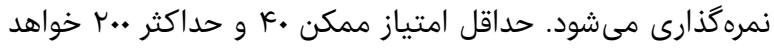

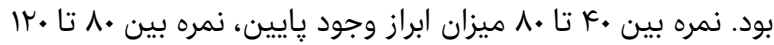

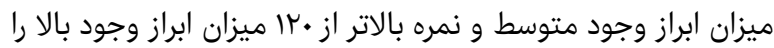

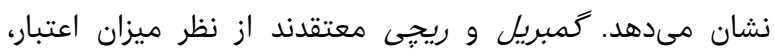

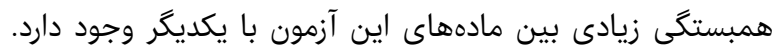

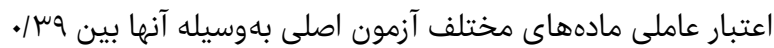

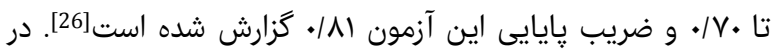

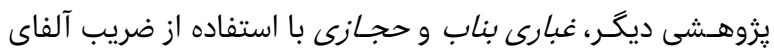

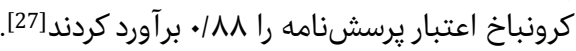

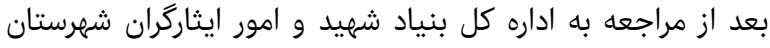

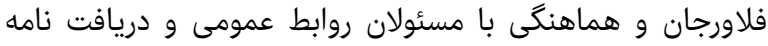

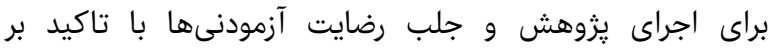

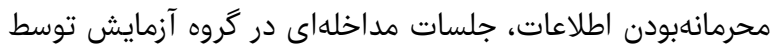

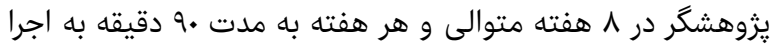

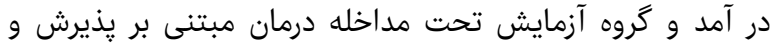

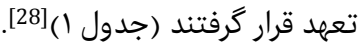

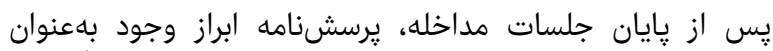

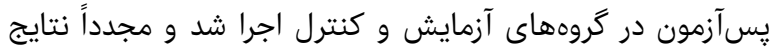

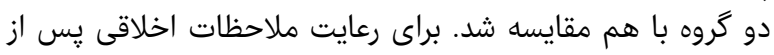

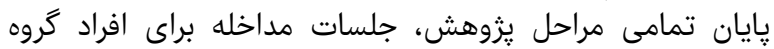

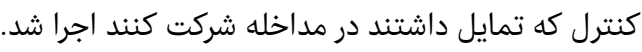

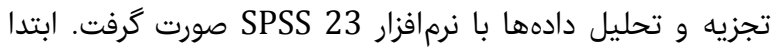

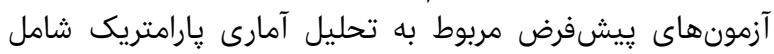

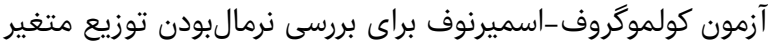

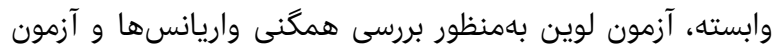




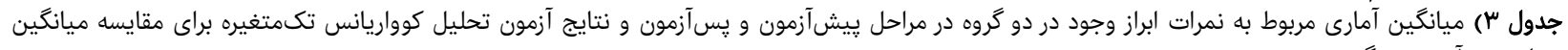

\begin{tabular}{|c|c|c|c|c|c|c|c|}
\hline سطح معنىدارى & توان آمارى & مجذور اتا & Fقدار F مق F & درجه آزادى & يسآزمون & ييش آزمون & كروهها \\
\hline.$|.|$. &.$/ 998$ & - / kqr & rH/Re. & 1 & $101 / 1 \mu_{ \pm}+r / \mu \Lambda$ & $1.9 / 9 \mu \pm I V / 1$. & كروه آزمايش \\
\hline 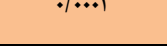 & $\cdot / 77\rangle$ & . 1Tया & ristry. & 1 & $11 \mu / \| \mu \pm \mu r / q$. & $\mid r \cdot / r \cdot \pm \backslash \Lambda / \Lambda \Lambda$ & گروه كنترل \\
\hline
\end{tabular}

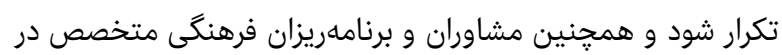

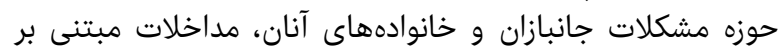

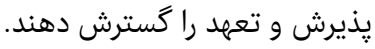

\section{نتيجه}

درمان مبتنى بر يذيرش و تعهد مئى دانواند بر ابراز وجود دختران

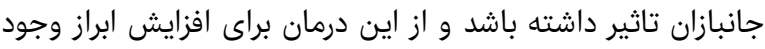

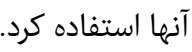

تشكر و قدردانى: از همكارى و مساعدت مسئولان بنياد شهيد و وخان

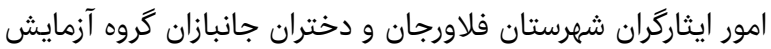

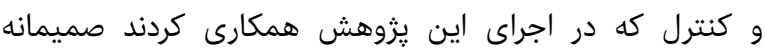

قدردانى مى شود.

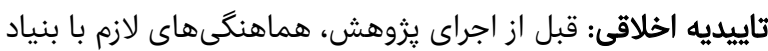

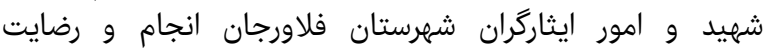

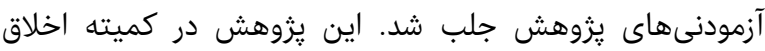

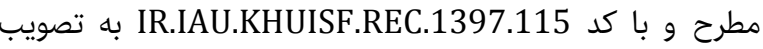

تعارض منافع: تعارض منافعى وجود ندارد.

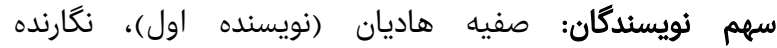

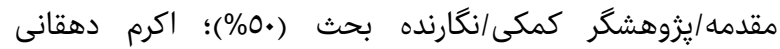

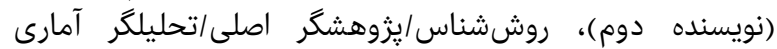

منابع مالى: اعتبار مالى اين يزوهش توسط نويسنده اول تامين

1- Friedman MJ. Veterans' mental health in the wake of war. N Engl J Med. 2005;352(13):1287-1290.

2- Eskandari H. The survey of veterans' depression and anxiety and its relationship with veterans' Social economic activities. J Milit Med. 2015;16(4):197-203. [Persian]

3- Mozafari MR, Ghanizadeh A, Ashkani H, Firoozabadi A, Alishahi MJ, Dehbozorgi GR, et al. The prevalence of anxiety and depression disorders in the children of veterans of Shiraz, Iran: A case control study. Qom Univ Med Sci J. 2009;3(2):19-24. [Persian]

4- Aghayousefi A, Sharif N. Personal well-being and stress symptoms in wives of Iranian martyrs, prisoners of wars and disabled veterans. Iran J Psychiatry.2010;5(1):28-34.

5- Ahmadzadeh G, Malekian A. Aggression, anxiety and social development in adolescent children of war veterans with PTSD versus those of nonveterans. Yafteh. 2004;5(19):63-9. [Persian]

6- Radfar S, Haghani H, Tavalaei SA, Modirian E, Falahati M. Evaluation of Mental health state in veterans family (15-18 Y/O adolescents). J Milit Med. 2005;7(3):203-9. [Persian]

7- Roshan Chesli R, Sharbati A, Rahimi Z. Comparison of high school students mental health in regard to generation and place of residence. J Train Learn Res.

مطالعه حاضر با هدف بررسى اثريذيرى درمان مبتنى بر يذيرش و دوان

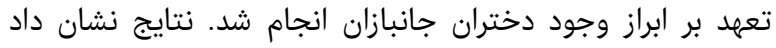

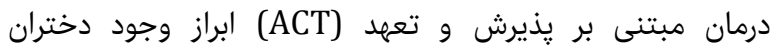

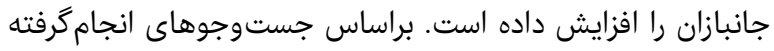

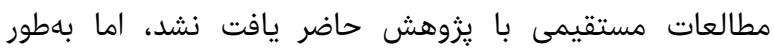

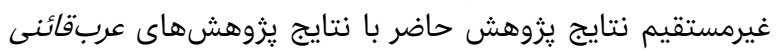

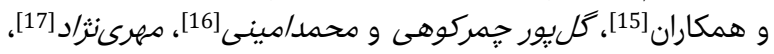

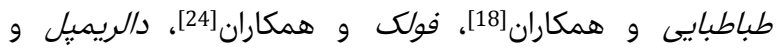

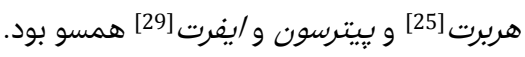

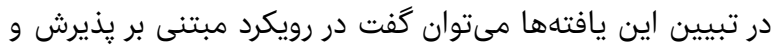

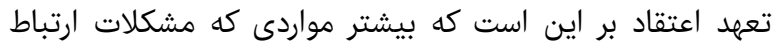

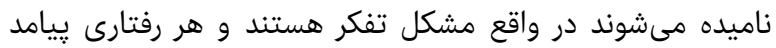

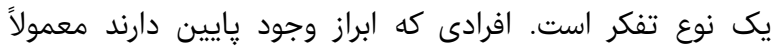

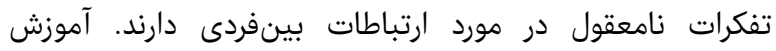

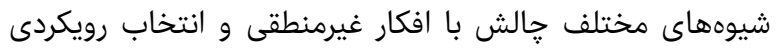

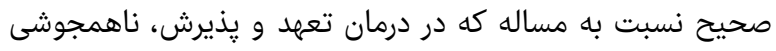

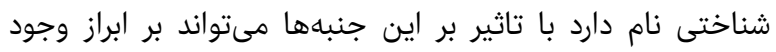

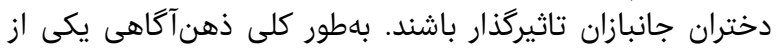

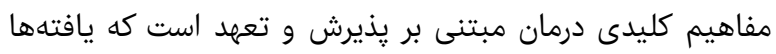

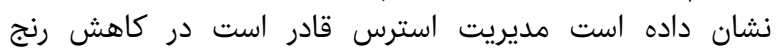

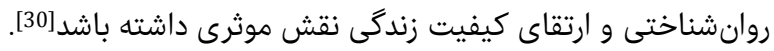

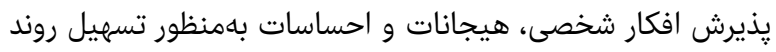

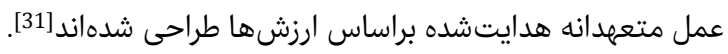

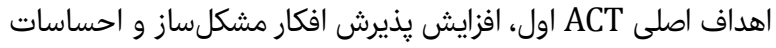

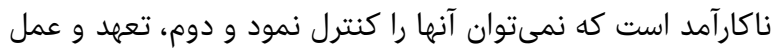

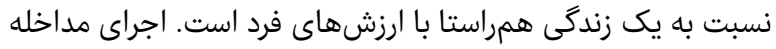

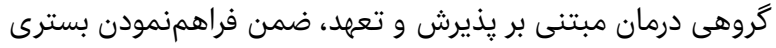

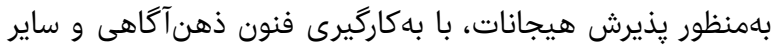

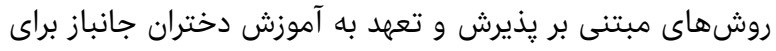

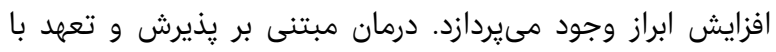

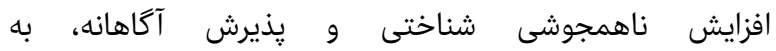

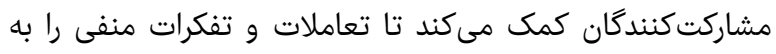

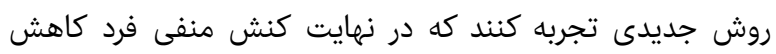

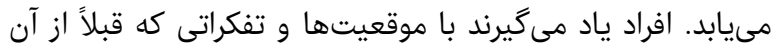

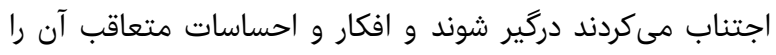

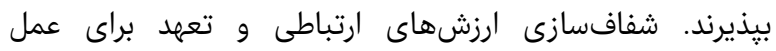

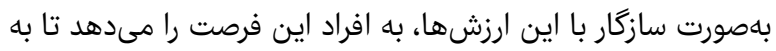

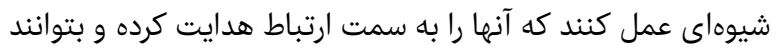
اضطراب فردى را كاهش دهند.

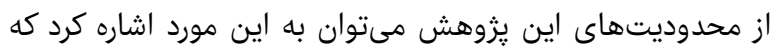

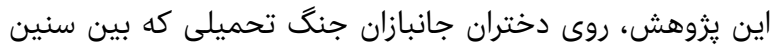

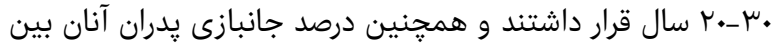

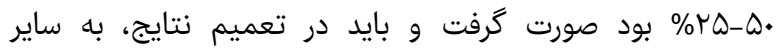

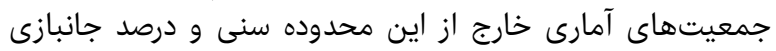
يدران، جوانب احتياط را رعايت كرد.

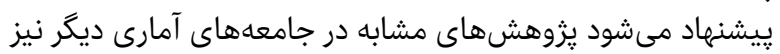




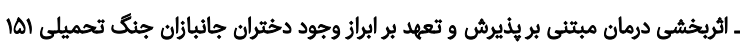

20- Howells A, Ivtzan I, Eiroa-Orosa FJ. Putting the 'app' in happiness: a randomized controlled trial of a smartphone-based mindfulness intervention to Enhance Wellbeing. J Happiness Stud. 2016;17(1):163-85.

21- Hor M, Aghaei A, Abedi A, Attari A. The effectiveness of acceptance and commitment therapy on depression in patients with type 2 diabetes. J Res Behav Sci. 2013;11(2):121-8. [Persian]

22- Anvari MH, Ebrahimi A, Taher Neshatdoust H, Afshar $\mathrm{H}$, Abedi A. The effectiveness of group-based acceptance and commitment therapy on pain-related anxiety, acceptance of pain and pain intensity in patients with chronic pain. J Isfahan Med Sch. 2014;32(295):1156-65. [Persian]

23- Bohlmeij ET, Lamers SM, Fledderus M. Flourishing in people with depressive symptomatology increases with acceptance and commitment therapy. post-hoc analyses of a randomized controlled trial. Behav Res Ther. 2015;65:101-6.

24- Folk F, Parling T, Melin L. Acceptance and commitment therapy for depression: a preliminary randomized clinical trial for unemployed on long -term sick leave. Cogn Behav Pract. 2012;19(4):583-94.

25- Dalrymple KL, Herbert JD. Acceptance and commitment therapy for generalized social anxiety disorder: a pilot study. Behav Modif. 2007;31(5):543-68. 26- Gambrill ED, Richey CA. An assertion inventory for use in assessment and research. Behav Ther. $1975 ; 6(4) ; 550-61$.

27- Ghobari Bonab B, Hejazi M. Relationship among assertiveness, self-esteem and academic achievement in gifted and regular school students. J Excep Child. 2007;7(3):299-316. [Persian]

28- Honarparvaran N, Mirzaei Kia H, Nayeri A, Lotfi M. Practical guide for therapists in acceptance and commitment therapy. 1st Edition. Mashhad: Omid-e Mehr; 2014. [Persian]

29- Peterson BD, Eifert GH. Using acceptance and commitment therapy to treat infertility stress. Cogn Behav Pract. 2011;18(4):577-87.

30- Hayes SC. Acceptance and commitment therapy, relational frame theory, and the third wave of behavioral and cognitive therapies. Behav Ther. 2004;35(4):639-65. 31- Hayes SC, Wilson KG, Gifford EV, Bissett R, Piasecki M, Batten SV, et al. A preliminary trial of twelve-step facilitation and acceptance and commitment therapy with polysubstance-abusing methadone-maintained opiate addicts. Behav Ther. 2004;35(4):667-88.
2011;1(45):71-8. [Persian]

8- Triplett R, Payhe B. Problem solving as reinforcement in adolescent drug use: Implication for theory and policy. J crim Justice. 2004;32(6):617-30.

9- Pirsaghi F, Nazari AM, Hajihassani M, Nadalipour H. The effect of self-differentiation therapy on assertiveness of female students of Allameh Tabataba'i University. Clin Psychol Stud. 2014;4(16):19-34. [Persian]

10- Stafford-Brown J, Pakenham KI. The effectiveness of an ACT informed intervention for managing stress and improving therapist qualities in clinical psychology trainees. J Clin Psychol. 2012;68(6):592-613.

11- Ezadi R, Abedi MR. Acceptance and commitment therapy. 3rd Edition. Tehran: Jangal publishing; 2014. [Persian]

12- Twohig MP. The application of acceptance and commitment therapy to obsessive-compulsive disorder. Cogn Behave Pract. 2009;16(1):18-28.

13- Smout MF, Longo M, Harrison S, Minniti R, Wickes W, White JM. Psychosocial treatment for methamphetamine use disorders: a preliminary randomized controlled trial of cognitive behavior therapy and acceptance and commitment therapy. Subst Abus. 2010;31(2):98-107.

14- Fennell MJ. Depression, low self-esteem and mindfulness. Behav Res Ther. 2004;42(9):1053-67.

15- Arab Ghaeni M, Hashemian K, Mojtabaei M , Majdara E, Aghabeiki A. The effect of mindfulness training (MBSR) on the increasing assertiveness among anxious students. Urmia Med J. 2017;28(2):119-29. [Persian]

16- Golpour Chamarkouhi R, Mohammadamini Z. The efficacy of mindfulness based stress reduction on mindfulness and assertiveness of students with test anxiety. J Sch Psychol. 2012;1(3):82-100. [Persian]

17- Mehrinejad SA. Investigating the effectiveness of mindfulness-based cognitive therapy to reduce shyness and increase the assertiveness puberty girls. Clin Psychol Stud. 2018:8(29);97-111. [Persian]

18- Tabatabaei A, Sajjadian I, Motamedi M. Effectiveness of acceptance and commitment therapy on sexual function, sexual self-consciousness and sexual assertiveness among women with sexual dysfunction. J Res Behav Sci. 2017;15(1):84-92. [Persian]

19- Izadi R, Taher Neshatdoiust H, Asgari K, Abedi MR. Comparison of the efficacy of acceptance and commitment therapy and cognitive-behavior therapy on symptoms of treatment of patients with obsessivecompulsive disorder. J Res Behav Sci. 2014;12(1):19-33. [Persian] 